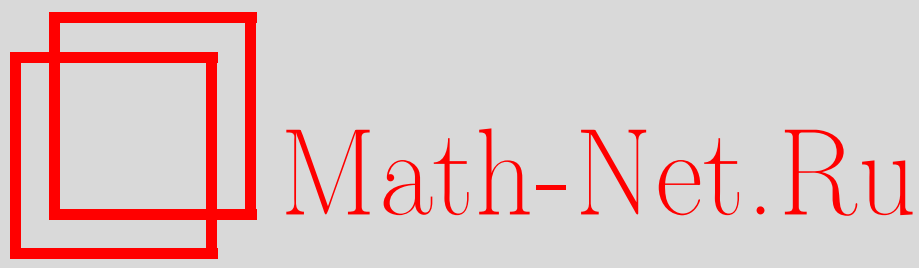

Д. Х. Зайнетдинов, Предельно монотонная сводимость множеств и $\Sigma$ определимость абелевых групп, Изв. вузов. Матем., 2019, номер 2, 21-28

DOI: https://doi.org/10.26907/0021-3446-2019-2-21-28

Использование Общероссийского математического портала Math-Net.Ru подразумевает, что вы прочитали и согласны с пользовательским соглашением

http://www . mathnet.ru/rus/agreement

Параметры загрузки:

IP: 54.174 .149 .18

26 апреля 2023 г., 18:29:37 
Известия вузов. Математика 2019, № 2, c. 21-28 https://kpfu.ru/science/nauchnye-izdaniya/ivrm/

e-mail: izvuz.matem@kpfu.ru

Д.Х. ЗАЙНЕТДИНОВ

\title{
ПРЕДЕЛЬНО МОНОТОННАЯ СВОДИМОСТЬ МНОЖЕСТВ И ऽ-ОПРЕДЕЛИМОСТЬ АБЕЛЕВЫХ ГРУПП
}

\begin{abstract}
Аннотация. Работа посвящена изучению предельно монотонных множеств, а также исследованию основных структурных свойств предельно монотонной сводимости (для краткости будем записывать также $l m$-сводимость) между множествами. В работе получено описание алгоритмической зависимости между предельно монотонной сводимостью множеств, определенной в терминах $\Sigma$-сводимости семейств начальных сегментов, и $\Sigma$-определимостью абелевых групп.
\end{abstract}

Ключевые слова: предельно монотонная функция, предельно монотонное множество, предельно монотонная сводимость, семейство подмножеств натуральных чисел, $\Sigma$-сводимость, $\Sigma$-определимость, абелева группа, наследственно конечная надстройка.

УДК: 510.5

DOI: $10.26907 / 0021-3446-2019-2-21-28$

\section{1. ПРЕДВАРИТЕЛЬНЫЕ СВЕДЕНИЯ}

Одна из методологических проблем современной теории моделей направлена на то, чтобы оценить сложность исследуемого объекта таким образом, чтобы представленная алгебраическая структура наследовала эффективные свойства этого объекта. К одному из таких объектов исследования относится класс неубывающих по последнему аргументу вычислимых трехместных функций. С помощью этих функций Н.Г. Хисамиевым [1] был доказан критерий конструктивизируемости прямой суммы циклических $p$-групп.

Определения предельно монотонных функций и предельно монотонных множеств, которые используются в данной статье, можно найти в работе [2]. Основные сведения, полученные при изучении предельно монотонных функций, множеств и последовательностей можно найти в работах [2]-[4]. Кроме того, с несколькими интересными результатами, касающимися вопросов предельно монотонной сводимости $\Sigma_{2}^{0}$-множеств, можно ознакомиться в работе [5]. По части основных обозначений, встречающихся в настоящей статье, будем придерживаться работ [6] и [7].

И.Ш. Калимуллиным и В.Г. Пузаренко в работе [7] вводится понятие $\Sigma$-сводимости на семействах подмножеств натуральных чисел, согласованное с понятием $\Sigma$-определимости в допустимых множествах и позволяющее рассматривать семейство само по себе, не фиксируя при этом его представление с помощью натуральных чисел.

Поступила в редакцию 02.10.2017, после доработки 22.05.2018. Принята к публикации 26.09.2018

Благодарности. Работа выполнена за счет средств субсидии, выделенной Казанскому федеральному университету для выполнения государственного задания в сфере научной деятельности (проект № 1.1515.2017/4.6). 
Определение 1. Пусть дано семейство $\mathcal{S} \subseteq 2^{\mathbb{N}}$. $\mathcal{S}$-кортежем называется множество вида $\{\langle n, k\rangle\} \oplus\left(X_{1} \oplus \cdots \oplus X_{n}\right)$, где $n, k \in \mathbb{N}$ и $X_{i} \in \mathcal{S}$ для всех $i=\overline{1, n}$. Через $K_{\mathcal{S}}$ будем обозначать множество всех $\mathcal{S}$-кортежей.

Определение 2. Семейство $\mathcal{S}_{0} \subseteq 2^{\mathbb{N}} \Sigma$-сводится к семейству $\mathcal{S}_{1} \subseteq 2^{\mathbb{N}}$ (обозначается как $\left.\mathcal{S}_{0} \sqsubseteq \Sigma \mathcal{S}_{1}\right)$, если

$$
\mathcal{S}_{0} \cup\{\emptyset\}=\left\{\Phi\left(X \oplus Y \oplus E\left(\mathcal{S}_{1}\right)\right) \mid X \in K_{\mathcal{S}_{1}}\right\}
$$

для некоторых оператора перечисления $\Phi$ и множества $Y \in K_{\mathcal{S}_{1}}$.

Здесь $E(\mathcal{S})=\left\{n \in \mathbb{N} \mid(\exists F \in \mathcal{S})\left[D_{n} \subseteq F\right]\right\}$, через $D_{n}$ обозначаем конечное множество с каноническим индексом $n$.

Определим для данных множеств $A, B \subseteq \mathbb{N}$ семейства начальных сегментов $\mathcal{S}(A)=$ $\{\mathbb{N} \uparrow a: a \in A\}$ и $\mathcal{S}(B)=\{\mathbb{N} \uparrow b: b \in B\}$ соответственно. Доказательство следующего результата, устанавливающего алгоритмическую зависимость между понятиями $l \mathrm{~m}$-сводимости множеств и $\Sigma$-сводимости семейств начальных сегментов, можно найти в работе [8].

Теорема 1. Утверждение $A \leqslant_{l m} B$ имеет место тогда и толъко тогда, когда $\mathcal{S}(A) \sqsubseteq_{\Sigma}$ $\mathcal{S}(B)$.

Рассмотрим теорию Крипке-Платека с праэлементами $\left(\mathbf{K P U}_{\sigma}\right)$ для произвольной сигнатуры $\sigma$, расширяющей сигнатуру $\sigma_{1}=\left\{\emptyset ; \in^{2}\right\}$. Определение аксиом данной теории можно найти, например, в [9]. Данная теория является слабым фрагментом теории ЦермелоФренкеля $(\mathbf{Z F})$ с праэлементами, позволяющая иметь дело с ординалами, которые определяются аналогично тому, как и в теории ZF. В структуре $\mathfrak{M}=\left\langle A, \emptyset^{A}, \epsilon^{A}, \ldots\right\rangle$ этой теории элементы основного множества $A$ разбиваются на два непересекающихся класса $S(A)$ и $U(A)$, т. е. $A=S(A) \cup U(A)$ и $S(A) \cap U(A)=\emptyset$. Элементы из $S(A)$ называются "множествами", а элементы из $U(A)$ "праэлементами".

Под допустимым множеством будем понимать KPU-модель, в которой отношение принадлежности $(\in)$ вполне упорядочивает семейство всех ординалов данной модели.

Допустимые множества обозначаются через $\mathbb{A}, \mathbb{B}$, а их носители - через $A, B$ соответственно. Структуры обозначаем через $\mathfrak{A}, \mathfrak{B}, \ldots$ Отметим, что любое допустимое множество содержит в качестве подмножества множество натуральных ординалов, на котором можно задать структуру натуральных чисел.

Пусть $\sigma$ - сигнатура, которая включает символ равенства. Ниже приведены определения $\Delta_{0^{-}}, \Sigma$ - и П-формул сигнатуры $\sigma \cup\{\in, \ldots\}$.

Определение 3. Класс $\Delta_{0}$ - формул сигнатуры $\sigma \cup\{\in, \ldots\}$ - наименьший класс, содержащий все атомарные формулы сигнатуры $\sigma \cup\{\in, \ldots\}$ и замкнутый относительно $\wedge, \vee, \rightarrow$, $\neg, \exists x \in t \forall x \in t$, где $x$ - переменная, а $t-$ терм, в котором $x$ не встречается $(\exists x \in t \ldots$ и $\forall x \in t \ldots$ служат сокращением для $\exists x(x \in t \wedge \ldots)$ и $\forall x(x \in t \rightarrow \cdots)$ соответственно).

Определение 4. Класс $\Sigma$ - (П-) формул сигнатуры $\sigma \cup\{\in, \ldots\}$ - наименьший класс, содержащий все $\Delta_{0}$-формулы сигнатуры $\sigma \cup\{\in, \ldots\}$ и замкнутый относительно $\wedge, \vee, \exists x \in t$ $\forall x \in t$, а также $\exists(\forall) x$, где $x$ - переменная, а $t$ - терм, в котором $x$ не встречается.

Пусть $\mathbb{A}$ - допустимое множество и $n \geqslant 1$.

Определение 5. $n$-местный предикат $R$ на $\mathbb{A}$ называется $\Sigma$-предикатом, если он определим некоторой $\Sigma$-формулой (возможно, с параметрами); $\Delta$-предикатом, если $R$ и $A^{n} \backslash R$ являются $\Sigma$-предикатами. 
Одноместные $\Sigma$ - и $\Delta$-предикаты будем называть $\Sigma$ - и $\Delta$-подмножествами соответственно. Частичная операция на $\mathbb{A}$ называется $\Sigma$-функиией, если ее график является $\Sigma$-предикатом.

Одним из важнейших классов допустимых множеств являются наследственно конечные надстройки. В данной работе будем рассматривать наследственно конечные надстройки над структурами, т. е. наименьшие по включению допустимые множества, которые содержат (как подмножества) в качестве праэлементов носители рассматриваемых структур.

Пусть $\mathfrak{M}=\langle M, \ldots\rangle-$ алгебраическая структура с носителем $M$ и некоторой предикатной сигнатурой $\sigma$ и $\sigma \cap\left\{\emptyset, \in^{2}, U^{1}\right\}=\emptyset$. Рассмотрим элементы из основного множества $M$ как праэлементы и определим индукцией следующую последовательность множеств над $M$

$$
H F_{0}(M), H F_{1}(M), H F_{2}(M), \ldots, H F_{n}(M), \ldots
$$

для всех натуральных $n \in \mathbb{N}$ по правилу

$$
\begin{gathered}
H F_{0}(M)=M, \\
H F_{n+1}(M)=H F_{n}(M) \cup \mathcal{P}_{\omega}\left(H F_{n}(M)\right),
\end{gathered}
$$

где $\mathcal{P}_{\omega}(M)$ - множество всех конечных подмножеств множества $M$.

Пусть

$$
H F(M) \rightleftharpoons \cup_{n \in \mathbb{N}} H F_{n}(M)
$$

Тогда $H F(M)$ - семейство всех наследственно конечных множеств; элементы из $H F(M)$ называются наследственно конечными множествами над $M$. На $H F(M)$ определим естественным образом структуру $\mathbb{H} \mathbb{F}(\mathfrak{M})$ сигнатуры $\sigma^{\prime}=\sigma \cup\left\{\emptyset, \in^{2}, U^{1}\right\}$, называемую наследственно конечной надстройкой над структурой $\mathfrak{M}$, причем $U^{\mathbb{H} \mathbb{F}(\mathfrak{M})}=M$.

Пусть $\mathfrak{A}$ - структура с носителем, являющимся подмножеством $A$ множества $H F(M)$. Каждой формуле $\Psi\left(a_{1}, \ldots, a_{n}\right)$ сигнатуры структуры $\mathfrak{A}$ с параметрами $a_{1}, \ldots, a_{n} \in A$, имеющей гёделев номер $m \in \mathbb{N}$, можно сопоставить элемент $\left(m,\left(a_{1}, \ldots\left(a_{n-1}, a_{n}\right) \ldots\right)\right)$ множества $H F(M)$. Здесь $(x, y)=\{\{x\},\{x, y\}\}$. Следовательно, под атомной диаграммой структуры $\mathfrak{A}$ будем понимать некоторое подмножество $H F(M)$.

Определение 6. Будем говорить, что алгебраическая структура $\mathfrak{A} \Sigma$-определима в наследственно конечной надстройке $\mathbb{H} \mathbb{F}(\mathfrak{M})$ (и записывать $\mathfrak{A} \leqslant \Sigma \mathbb{H} \mathbb{F}(\mathfrak{M})$ ), если

1) существует изоморфная копия структуры $\mathfrak{A}$, носитель который является подмножеством $H F(M)$;

2) атомная диаграмма структуры $\mathfrak{A}$ определима в $\mathbb{H} \mathbb{F}(\mathfrak{M})$ с помощью некоторой $\Sigma$-формулы.

В следующей теореме приводится описание $\Sigma$-сводимости семейств подмножеств натуральных чисел (см. определение 2) в терминах операторов перечисления.

Теорема 2 ([7]). Для семейств $\mathcal{S}_{0}$ и $\mathcal{S}_{1}$ следующие условия эквивалентны:

1) $\mathcal{S}_{0} \sqsubseteq \Sigma \mathcal{S}_{1}$

2) $\mathcal{S}_{0}$ вычислимо в $\mathbb{H} \mathbb{F}\left(\mathfrak{M}_{\mathcal{S}_{1}}\right)$ для структуры $\mathfrak{M}_{\mathcal{S}_{1}}$;

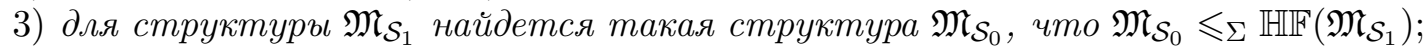

4) для любой структуры $\mathfrak{M}_{\mathcal{S}_{1}}$ найдется такая структура $\mathfrak{M}_{\mathcal{S}_{0}}$, что

$$
\mathbb{H} \mathbb{F}\left(\mathfrak{M}_{\mathcal{S}_{0}}\right) \sqsubseteq \Sigma \mathbb{H} \mathbb{F}\left(\mathfrak{M}_{\mathcal{S}_{1}}\right) ;
$$

5) для любого допустимого множества $\mathbb{A}$ из вычислимости $\mathcal{S}_{1}$ в $\mathbb{A}$ следует существование структуры $\mathfrak{M}_{\mathcal{S}_{0}}$ такой, что $\mathfrak{M}_{\mathcal{S}_{0}} \leqslant \Sigma \mathbb{A} ;$

6) для любого допустимого множества $\mathbb{A}$ из вычислимости $\mathcal{S}_{1}$ в $\mathbb{A}$ следует существование структуры $\mathfrak{M}_{\mathcal{S}_{0}}$ такой, что $\mathbb{H} \mathbb{F}\left(\mathfrak{M}_{\mathcal{S}_{0}}\right) \sqsubseteq \Sigma \mathbb{A}$. 
Стоит отметить, что в [10] исследована также $\Sigma$-определимость в наследственно конечных надстройках над алгебраическими структурами. В данной работе будем использовать следующий результат.

Предложение 1 ([11]). Пусть $\mathfrak{M}$ - структура конечной сигнатуры и пусть $\mathbb{A}$ - допустимое множество. Тогда $\mathfrak{M} \leqslant \Sigma \mathbb{A}$, если и только если $\mathbb{H} \mathbb{F}(\mathfrak{M}) \sqsubseteq_{\Sigma} \mathbb{A}$.

\section{2. $\Sigma$-ОПРЕДЕЛИМОСТЬ АБЕЛЕВЫХ ГРУПП И $l m$-СВОДИМОСТЬ МНОЖЕСТВ}

Пусть дано множество $A \subseteq \mathbb{N}$. Рассмотрим абелеву группу $G(A)$ следующего вида:

$$
G(A)=\bigoplus_{n \in A}\left(\bigoplus_{m \in \mathbb{N}} \mathbb{Z}_{p^{n}}\right),
$$

где $p$ принадлежит множеству простых чисел.

Перед тем как приступить к доказательству основного результата данной работы, установим справедливость следующей теоремы.

Теорема 3. Пусть дано множество $A \subseteq \mathbb{N}$. Пусть $\mathcal{S}(A)=\{\mathbb{N} \uparrow n: n \in A\}-$ семейство начальных сегментов и $G(A)=\bigoplus_{n \in A}\left(\bigoplus_{m \in \mathbb{N}} \mathbb{Z}_{p^{n}}\right)$ - абелева группа, определенные для множества $A$. Тогда $\mathcal{S}(A) \leqslant \Sigma \mathbb{H} \mathbb{F}(G(A))$, т.е. семейство $\mathcal{S}(A) \Sigma$-определимо в наследственно конечной надстройке $\mathbb{H} \mathbb{F}(G(A))$ над группой $G(A)$.

Доказательство. Покажем, что $\mathcal{S}(A) \leqslant \Sigma \mathbb{H} \mathbb{F}(G(A))$. Отметим, что $\Sigma$-определимость семейства $\mathcal{S}(A)$ в $\mathbb{H} \mathbb{F}(G(A))$ означает, что на некотором $\Sigma$-подмножестве из наследственно конечной надстройки $\mathbb{H} \mathbb{F}(G(A)$ ) (используя $\Sigma$-формулы) можно построить изоморфную копию семейства $\mathcal{S}(A)$. При исследовании $\Sigma$-определимости на семействах подмножеств натуральных чисел будем придерживаться работы [7], в которой получено соответствие, позволяющее отождествлять семейства с моделями.

По заданной группе $G(A)$ нетрудно построить над ней наследственно конечную надстройку $\mathbb{H} \mathbb{F}(G(A))$. Всякий элемент наследственно конечной надстройки $\mathbb{H} \mathbb{F}(G(A))$ можно записать как значение некоторого терма от праэлементов (элементов группы $G(A)$, которые в доказательстве теоремы будем обозначать через $u$ ), составленного из символов пустого множества $\varnothing$, объединения $\cup$, фигурных скобок $\{$ и $\}$. Элементы $u \in G(A)$ можно представить в виде

$$
u=x_{1} \oplus x_{2} \oplus \cdots \oplus x_{r}, \quad \text { где } x_{i} \in \mathbb{Z}_{p^{n_{i}}} \text { для } 1 \leqslant i \leqslant r, \quad r \in \mathbb{N} .
$$

Пусть $n=\min _{1 \leqslant i \leqslant r}\left\{n_{i}\right\}$. Среди элементов $u \neq 0$ находим такие, что $p u=0$ для некоторого простого числа $p$. Далее пытаемся максимально продолжить разложение найденных элементов $u$. С помощью данного процесса определяем множество $X_{u}=\left\{k \mid p^{k-1}\right.$ делит $\left.u\right\}$. При этом заметим, что для перечисления элементов множества $X_{u}$ использовали $\Sigma$-формулу: $\exists b\left(u=p^{k-1} b\right)$.

Покажем, что $\mathcal{S}(A)=\left\{X_{u} \mid p u=0, u \neq 0 \& u \in G(A)\right\}$.

1) Пусть $n \in \mathcal{S}(A)$. Покажем, что $n \in X_{u}$. Возьмем такой элемент $u \neq 0$, что $u=$ $p^{n-1} 1_{\mathbb{Z}_{p^{n}}} \in \mathbb{Z}_{p^{n}}$. Тогда $p u=p\left(p^{n-1} 1_{\mathbb{Z}_{p^{n}}}\right)=p^{n} 1_{\mathbb{Z}_{p^{n}}}=0$ в группе $\mathbb{Z}_{p^{n}}$.

Осталось установить, что множество $X_{u}$ не содержит элементов $m>n$. Предположим противное, а именно, что $u=p^{m-1} a=p^{n}\left(p^{m-n-1} a\right)=0$ в группе $\mathbb{Z}_{p^{n}}$. Получили противоречие с тем, что $u \neq 0$. Поэтому $X_{u}=\{1,2, \ldots, n\}$. 
2) Пусть $u \in G(A)$, где $G(A)=\bigoplus_{n \in A}\left(\bigoplus_{m \in \mathbb{N}} \mathbb{Z}_{p^{n}}\right)$ и $u=x_{1} \oplus x_{2} \oplus \cdots \oplus x_{r}$, где $x_{i} \in \mathbb{Z}_{p^{n_{i}}}$ для $1 \leqslant i \leqslant r, r \in \mathbb{N}$. Тогда

$$
u=p^{n-1} a=\sum_{i=1}^{r} p^{n_{i}-1} a_{i}=p^{\min \left\{n_{i}\right\}-1}\left(\sum_{i=1}^{r} p^{n_{i}-\min \left\{n_{i}\right\}} a_{i}\right),
$$

$n=\min _{1 \leqslant i \leqslant r}\left\{n_{i}\right\}$ и $p u=0$ в группе $\mathbb{Z}_{p^{n}}$. Так как $p^{n-1}$ делит $u$, то перечисляем элементы $1,2, \ldots, n$. При этом $\{1,2, \ldots, n\}=\{\mathbb{N}\lceil n: n \in A\}$.

Следовательно, установили, что $\mathcal{S}(A)=\left\{X_{u} \mid p u=0, u \neq 0 \& u \in G(A)\right\}$.

Таким образом, на $\Sigma$-подмножестве из наследственно конечной надстройки $\mathbb{H} \mathbb{F}(G(A))$, используя $\Sigma$-формулу, построили изоморфную копию семейства $\mathcal{S}(A)$. Отсюда непосредственно вытекает $\Sigma$-определимость семейства $\mathcal{S}(A)$ в наследственно конечной надстройке $\mathbb{H} \mathbb{F}(G(A))$.

Перейдем теперь непосредственно к доказательству основного результата данной статьи.

Теорема 4. Пусть $A, B \subseteq \mathbb{N}$. Пусть $G(A)=\bigoplus_{n \in A}\left(\bigoplus_{m \in \mathbb{N}} \mathbb{Z}_{p^{n}}\right) u G(B)=\bigoplus_{k \in B}\left(\bigoplus_{m \in \mathbb{N}} \mathbb{Z}_{p^{k}}\right)$ абелевы группы, определенные для множеств $A$ и $B$ соответственно. Тогда $A \leqslant_{\operatorname{lm}} B$, если и только если группа $G(A)$ ¿-определима в наследственно конечной надстройке $\mathbb{H} \mathbb{F}(G(B))$.

Доказательство. Необходимость. Рассмотрим для множеств $A, B \subseteq \mathbb{N}$ семейства начальных сегментов вида $\mathcal{S}(A)=\{\mathbb{N} \uparrow n: n \in A\}$ и $\mathcal{S}(B)=\{\mathbb{N} \uparrow k: k \in B\}$. Воспользуемся следующей цепочкой соотношений:

$$
A \leqslant_{l m} B \Longrightarrow \mathcal{S}(A) \sqsubseteq_{\Sigma} \mathcal{S}(B) \Longrightarrow \mathfrak{M}_{\mathcal{S}(A)} \leqslant_{\Sigma} \mathbb{H} \mathbb{F}\left(\mathfrak{M}_{\mathcal{S}(B)}\right) .
$$

В соотношении (1) первая импликация верна в силу теоремы 1, а вторая импликация следует из теоремы 2 в части, касающейся эквивалентности условий 1) и 3).

Воспользовавшись эквивалентностью между условиями 3) и 4) теоремы 2, заметим, что

$$
\mathfrak{M}_{\mathcal{S}(A)} \leqslant_{\Sigma} \mathbb{H} \mathbb{F}\left(\mathfrak{M}_{\mathcal{S}(B)}\right) \Longleftrightarrow \mathbb{H} \mathbb{F}\left(\mathfrak{M}_{\mathcal{S}(A)}\right) \sqsubseteq \Sigma \mathbb{H} \mathbb{F}\left(\mathfrak{M}_{\mathcal{S}(B)}\right)
$$

Применяем условие теоремы 3 к семейству $\mathcal{S}(B)$ и группе $G(B)$. Получим, что семейство $\mathcal{S}(B) \Sigma$-определимо в наследственно конечной надстройке $\mathbb{H} \mathbb{F}(G(B))$, т. е.

$$
\mathcal{S}(B) \leqslant_{\Sigma} \mathbb{H} \mathbb{F}(G(B)) .
$$

Применим к отношению (3) предложение 1. Тогда

$$
\mathbb{H} \mathbb{F}\left(\mathfrak{M}_{\mathcal{S}(B)}\right) \sqsubseteq_{\Sigma} \mathbb{H} \mathbb{F}(G(B)) .
$$

Отсюда с учетом правой части соотношения (2) имеем

$$
\mathbb{H} \mathbb{F}\left(\mathfrak{M}_{\mathcal{S}(A)}\right) \sqsubseteq \Sigma \mathbb{H} \mathbb{F}(G(B)) .
$$

Далее к отношению (5) применим эквивалентность условий 3) и 4) из теоремы 2. Получим

$$
\mathfrak{M}_{\mathcal{S}(A)} \leqslant \Sigma \mathbb{H} \mathbb{F}(G(B)) .
$$

Так как $\mathcal{S}(A) \leqslant_{\Sigma} \mathfrak{M}_{\mathcal{S}(A)}$, т. е. семейство $\mathcal{S}(A) \quad \Sigma$-определимо в $\mathfrak{M}_{\mathcal{S}(A)}$, то с учетом отношения (6) можно заключить, что

$$
\mathcal{S}(A) \leqslant_{\Sigma} \mathbb{H} \mathbb{F}(G(B)) .
$$

Таким образом, установили, что семейство $\mathcal{S}(A)$ - -определимо в наследственно конечной надстройке $\mathbb{H} \mathbb{F}(G(B))$. Для завершения доказательства теоремы в части необходимости осталось в отношении (7) сделать переход от семейства $\mathcal{S}(A)$ к группе $G(A)$ и затем найти $\Sigma$-определимое представление группы $G(A)$ в $\mathbb{H} \mathbb{F}(G(B))$. 
Используя конструкцию построения множества $X_{u}$ из доказательства теоремы 3 , определим множество $U_{b}=\left\{s \mid p^{s-1}\right.$ делит $\left.b\right\}$ для некоторого простого числа $p$. Для перечисления элементов множества $U_{b}$ использовали $\Sigma$-формулу $\Phi(s, b): \exists d\left(b=p^{s-1} d\right)$. Тогда по теореме 3 можно считать, что $\mathcal{S}(A)=\{\mathbb{N} \mid n: n \in A\}=\left\{U_{b} \mid b \in G(B)\right\}$. При этом элемент $s \in U_{b}$, если и только если справедлива $\Sigma$-формула $\Phi(s, b)$.

На элементах, принадлежащих наследственно конечной надстройке $\mathbb{H} \mathbb{F}(G(B)$ ), с помощью $\Sigma$-формулы строим универсум, который будет изоморфен группе $G(A)$. Для этого рассмотрим факторгруппу $\mathbb{Q} / \mathbb{Z} \subseteq \mathbb{H} \mathbb{F}(G(B))$. Определим группу $\mathbb{Q}[0,1)$ с операцией сложения: $\forall a, b \in \mathbb{Q}[0,1) a+b=a+b-[a+b]$, при этом $\mathbb{Q}[0,1) \cong \mathbb{Q} / \mathbb{Z}$. Отождествляем элементы группы $\mathbb{Q}[0,1)$ с их номерами в $\mathbb{N} \subseteq \mathbb{H} \mathbb{F}(\emptyset) \subseteq \mathbb{H} \mathbb{F}(G(B))$ относительно стандартного кодирования.

Каждому элементу $b$ из области определения наследственно конечной надстройки $\mathbb{H} \mathbb{F}(G(B))$ ставим в соответствие группу $G_{b}=\left\langle\left\{\frac{1}{p^{s}} \mid s \in U_{b}\right\}\right\rangle$, где $p$ - это некоторое простое число. Тогда элементы $\frac{1}{p^{s}} \in \mathbb{Q}[0,1)$, где $1 \leqslant s \leqslant n$ и $n \in A$. Поэтому $G_{b} \subseteq \mathbb{Q}[0,1)$. Отметим, что все элементы $\left\langle\frac{1}{p}, \frac{1}{p^{2}}, \frac{1}{p^{3}}, \ldots, \frac{1}{p^{n}}\right\rangle$ группы $G_{b}$ могут быть получены из элемента $\left\langle\frac{1}{p^{n}}\right\rangle$ путем домножения на $p^{s-1}$, где $1 \leqslant s \leqslant n$. Таким образом, $G_{b}$ является циклической группой с порождающим элементом $\left\langle\frac{1}{p^{n}}\right\rangle$.

Докажем, что $G_{b}=\left\langle\left\{\frac{1}{p^{s}} \mid s \in U_{b}\right\}\right\rangle$ изоморфна группе $\mathbb{Z}_{p^{n}}=\left\{1, p, \ldots, p^{n-1}\right\}$, где $n \in A$. Для этого определим биективную функцию $g: G_{b} \longrightarrow \mathbb{Z}_{p^{n}}$ по правилу

$$
g\left(\frac{1}{p^{s}}\right)=p^{s}, 1 \leqslant s \leqslant n .
$$

Таким образом, нашли представление группы

$$
G(A)=\bigoplus_{n \in A}\left(\bigoplus_{m \in \mathbb{N}} \mathbb{Z}_{p^{n}}\right) \cong \bigoplus_{b \in \mathbb{H} F\left(G_{b}\right)} G_{b}
$$

Элементами группы $\bigoplus_{b \in \mathbb{H}}\left(G_{b}\right) G_{b}$ являются функции $f: \mathbb{H} \mathbb{F}(G(B)) \longrightarrow \mathbb{Q}[0,1)$ с конечной областью определения. При этом

$$
f \in \bigoplus_{b \in \mathbb{H} \mathbb{F}\left(G_{b}\right)} G_{b} \Longleftrightarrow \forall b \in \operatorname{dom}(\mathbb{H} \mathbb{F}(G(B))) \exists l, s\left[f(b)=\frac{l}{p^{s}} \& \Phi(s, b)\right] .
$$

Получили $\Sigma$-формулу, описывающую графики функций $f$. Поэтому множество конечных графиков таких функций будет $\Sigma$-определимо в $\mathbb{H} \mathbb{F}(G(B))$. Следовательно, в $\mathbb{H} \mathbb{F}(G(B))$ будет также $\Sigma$-определима группа $\bigoplus_{b \in \mathbb{H} F\left(G_{b}\right)} G_{b}$, которая изоморфна $G(A)$.

Достаточность. Пусть группа $G(A) \Sigma$-определима в наследственно конечной надстройке $\mathbb{H} \mathbb{F}(G(B))$ над группой $G(B)$, т. е. выполняется отношение

$$
G(A) \leqslant \Sigma \mathbb{H} \mathbb{F}(G(B)) .
$$

Докажем, что $A \leqslant_{l m} B$. Отметим, что согласно теореме 1

$$
A \leqslant_{l m} B \Longleftrightarrow \mathcal{S}(A) \sqsubseteq_{\Sigma} \mathcal{S}(B) .
$$

Из теоремы 2 в части, касающейся эквивалентности условий 1) и 3), следует

$$
\mathcal{S}(A) \sqsubseteq_{\Sigma} \mathcal{S}(B) \Longleftrightarrow \mathfrak{M}_{\mathcal{S}(A)} \leqslant_{\Sigma} \mathbb{H} \mathbb{F}\left(\mathfrak{M}_{\mathcal{S}(B)}\right) .
$$

Таким образом, с учетом условия (9) достаточно показать, что

$$
\mathfrak{M}_{\mathcal{S}(A)} \leqslant \Sigma \mathbb{H} \mathbb{F}\left(\mathfrak{M}_{\mathcal{S}(B)}\right) .
$$


Поскольку семейство $\mathcal{S}(A) \quad \Sigma$-определимо в $\mathfrak{M}_{\mathcal{S}(A)}$, т. е. $\mathcal{S}(A) \leqslant \Sigma \mathfrak{M}_{\mathcal{S}(A)}$, то для доказательства $\Sigma$-определимости структуры $\mathfrak{M}_{\mathcal{S}(A)}$ в $\mathbb{H} \mathbb{F}\left(\mathfrak{M}_{\mathcal{S}(B)}\right)$ достаточно установить $\Sigma$-определимость семейства $\mathcal{S}(A)$ в $\mathbb{H} \mathbb{F}\left(\mathfrak{M}_{\mathcal{S}(B)}\right)$.

Покажем, что

$$
\mathcal{S}(A) \leqslant \Sigma \mathbb{H} \mathbb{F}\left(\mathfrak{M}_{\mathcal{S}(B)}\right) .
$$

Так как по условию теоремы $G(A) \leqslant \Sigma \mathbb{H} \mathbb{F}(G(B))$, то зная строение группы $G(A)$, нетрудно построить над ней наследственно конечную надстройку $\mathbb{H} \mathbb{F}(G(A))$. Тогда с учетом (8)

$$
\mathbb{H} \mathbb{F}(G(A)) \leqslant \Sigma \mathbb{H} \mathbb{F}(G(B)) .
$$

Далее из теоремы 3 имеем $\mathcal{S}(A) \leqslant \Sigma \mathbb{H} \mathbb{F}(G(A))$. Следовательно, с учетом (11) имеем

$$
\mathcal{S}(A) \leqslant_{\Sigma} \mathbb{H} \mathbb{F}(G(B)) .
$$

Для доказательства отношения (10) осталось установить, что $\mathbb{H} \mathbb{F}(G(B)) \leqslant_{\Sigma} \mathbb{H} \mathbb{F}\left(\mathfrak{M}_{\mathcal{S}(B)}\right)$. Так как $\mathcal{S}(B) \leqslant \Sigma \mathfrak{M}_{\mathcal{S}(B)}$, то используя конструкцию из доказательства теоремы в части необходимости, можем полагать, что $\mathcal{S}(B)=\left\{U_{b} \mid b \in \mathfrak{M}_{\mathcal{S}(B)}\right\}$. При этом $z \in U_{b}$, если и только если верна $\Sigma$-формула $\Phi(z, b)$.

Таким образом, для построения группы $G(B)$ применяем аналогичную конструкцию, как и при доказательстве теоремы в части необходимости. А именно, используя семейство $\mathcal{S}(B)$ и отношение $\mathcal{S}(B) \leqslant \Sigma \mathfrak{M}_{\mathcal{S}(B)}$, строим такую группу $G(B)$, что $G(B) \leqslant \Sigma \mathbb{H} \mathbb{F}\left(\mathfrak{M}_{\mathcal{S}(B)}\right)$. Далее, по группе $G(B)$ строим наследственно конечную надстройку $\mathbb{H} \mathbb{F}(G(B))$. Тогда получим

$$
\mathbb{H} \mathbb{F}(G(B)) \leqslant_{\Sigma} \mathbb{H} \mathbb{F}\left(\mathfrak{M}_{\mathcal{S}(B)}\right) .
$$

С учетом полученных выше соотношений имеем следующую цепочку:

$$
\mathcal{S}(A) \leqslant_{\Sigma} \mathbb{H} \mathbb{F}(G(A)) \leqslant_{\Sigma} \mathbb{H} \mathbb{F}(G(B)) \leqslant_{\Sigma} \mathbb{H} \mathbb{F}\left(\mathfrak{M}_{\mathcal{S}(B)}\right) .
$$

Поэтому из $(12)$ получим $\mathcal{S}(A) \leqslant \Sigma \mathbb{H} \mathbb{F}\left(\mathfrak{M}_{\mathcal{S}(B)}\right)$.

Таким образом, установили справедливость отношения (10), из которого, как показано выше, следует $A \leqslant \operatorname{lm} B$.

\section{ЛитературА}

[1] Хисамиев Н.Г. Критерий конструктивизируемости прямой суммы ииклических р-групп, Изв. АН Казахской ССР, сер. физ.-матем. 98 (1), 51-55 (1981).

[2] Khoussainov B., Nies A., Shore R. Computable models of theories with few models, Notre Dame J. Formal Logic 38 (2), 165-178 (1997).

[3] Kalimullin I., Khoussainov B., Melnikov A. Limitwise monotonic sequences and degree spectra of structures, Proc. Amer. Math. Soc. 141 (9), 3275-3289 (2013).

[4] Downey R.G., Kach A.M., Turetsky D. Limitwise monotonic functions and their applications, Proceedings of the 11th Asian Logic Conf. World Sci, 59-85 (2011).

[5] Зайнетдинов Д.Х., Калимуллин И.Ш. О пределъно монотонной сводимости $\Sigma_{2}^{0}$-множеств, Учен. зап. Казанск. гос. ун-та. Сер. Физ.-матем. науки 156 (1), 22-30 (2014).

[6] Faizrahmanov M., Kalimullin I., Zainetdinov D. Maximality and minimality under limitwise monotonic reducibility, Lobachevskii J. Math. 35 (4), 333-338 (2014).

[7] Калимуллин И.Ш., Пузаренко В.Г. О сводимости на семействах, Алгебра и логика 48 (1), 31-53 (2009).

[8] Зайнетдинов Д.Х. $\Sigma$-сводимость и lm-сводимость множеств и последовательностей множеств, Учен. зап. Казанск. ун-та. Сер. Физ.-матем. науки 158 (1), 51-65 (2016).

[9] Ершов Ю.Л. Определимость и вычислимость, 2-е изд., испр. и доп. (Экономика, М.; Научн. книга, Новосибирск, 2000).

[10] Пузаренко В.Г. О вычислимости над моделями разрешимых теорий, Алгебра и логика 39 (2), 170-197 (2000).

[11] Пузаренко В.Г. Об одной сводимости на допустимых множествах, Сиб. матем. журн. 50 (2), 415-429 (2009). 
Дамир Хабирович Зайнетдинов

Казанский федеральный университет,

ул. Кремлевская, д. 18, г. Казань, 420008, Россия,

e-mail : damir.zh@mail.ru

D.Kh. Zainetdinov

\section{Limitwise monotonic reducibility of sets and $\Sigma$-definability of abelian groups}

Abstract. The paper is devoted to the study of limitwise monotonic sets, as well as to the investigation of the main structural properties of limitwise monotonic reducibility (for short we will also write $l m$-reducibility) between sets. In this paper, we obtain a description of the algorithmic dependence between the limitwise monotonic reducibility of sets, which defined in terms of the $\Sigma$-reducibility of the families of initial segments, and the $\Sigma$-definability of abelian groups.

Keywords: limitwise monotonic function, limitwise monotonic set, limitwise monotonic reducibility, family of subsets of natural numbers, $\Sigma$-reducibility, $\Sigma$-definability, abelian group, hereditarily finite superstructure.

Damir Khabirovich Zainetdinov

Kazan Federal University,

18 Kremlyovskaya str., Kazan, 420008 Russia,

e-mail: damir.zh@mail.ru 